\title{
Not Hardwired: The Complex Neurobiology of Sex Differences in Violence
}

\author{
Debra Niehoff, PhD
}

\begin{abstract}
Behavior is the product of a brain shaped by a dynamic interaction between genetic and environmental factors. This neuroplasticity facilitates adaptation but can also lead to behavioral pathology when individuals with inherent vulnerabilities are exposed to dysfunctional environments, particularly early in life. Differences in the frequency and intensity of violent behavior in men and women may reflect underlying differences in brain structure, function, or connectivity that result from such interactions. This perspective provides a brief overview of research on sex differences in the neural circuitry mediating emotion, stress responses, and a specific gene-environment interaction, all of which may contribute to sex differences in violence.
\end{abstract}

\section{Introduction}

B EHAVIOR ORIGINATES IN THE BRAIN, and violence is no exception. Over the past several decades, neurobiological research has identified alterations in the neural circuitry underlying emotional regulation and in the neuroendocrine mechanisms mediating responses to stress that are characteristic of violent individuals. Furthermore, this research has described distinct neurobiological profiles for several patterns of violent behavior-most notably, reactive or hostile violence characterized by excessive emotional arousal and impulsive reactions to benign stimuli, and its mirror image, antisocial/psychopathic violence characterized by blunted emotional responses, indifference to risk, and a lack of empathy (Niehoff 1999; Davidson 2000; Haller 2005; Coccaro 2007; Glenn and Raine 2008).

Neurobiological explanations for behavior are essentially developmental explanations based on the recognition that brain structure and function are shaped by a dynamic interaction between genes and environmental factors. This interaction is reflected in environmentally induced changes at the cellular level, including gene expression, and is especially important during the more than two decades needed to complete brain maturation (Gogtay 2004; Curley 2011). Although the resulting plasticity is usually advantageous, enabling each individual to adapt to his or her unique circumstances, it can also be a liability when the environment is impoverished, dangerous, chaotic, or toxic, encoding exposure to these environmental insults in the form of maladaptive neural adaptations that can lead to dysfunctional behavior.

The biological mechanisms that enable this neuroplasticity are operative in both men and women. As a result, neither sex is immune to deleterious interactions between genetic and environmental factors that culminate in violent behavior. Nonetheless, rates of violence are disproportionately higher in men, a disparity that has been attributed to a wide range of sociocultural, psychological, and biological variables (Moffitt 2001; Bennett 2005). The neurodevelopmental perspective described above incorporates all of these in a holistic explanation, which proposes that familial, social, cultural, and educational influences, acting in concert with genes that determine individual sensitivity to these factors, are translated into modifications in brain structure, function, and connectivity. As a result, sex differences at the neural level may provide clues to sex differences in the propensity for violence.

Neurobiologists have documented numerous examples of sex differences in the human brain, including differences in brain regions and circuits relevant to violent behavior. In addition, research on the biological consequences of environmental influences has highlighted the important contribution of sex differences in vulnerability, resilience, and life experience to differences in behavior. Three examples of this research are summarized below. Over the coming months, future articles will examine these and other sex-related aspects of the neurobiology of violence in greater detail.

\section{The Anatomy of Emotion: Sex Differences in Neural Architecture and Their Relevance to Violence}

It has been clear for more than 100 years that different neural functions have different neuroanatomical origins. Early models of functional localization, based in large part on deficits after localized brain injury or electrical stimulation of discrete brain regions, emphasized the functional roles of individual brain regions. More recently, an improved

Department of Social and Behavioral Science, Bucks County Community College, Newtown, Pennsylvania. 
understanding of the anatomical connections between brain regions, patterns of brain activity observed during functional magnetic resonance imaging (fMRI), and correlations in the activity of multiple brain regions obtained through more detailed mathematical analysis of fMRI data have replaced this simplistic concept with a circuit model that attributes functionality to the coordinated effort of a network of brain regions (Stevenson 2012).

The network responsible for evaluating the emotional significance of a stimulus, selecting a response, and calibrating the magnitude of that response includes parts of the prefrontal cortex-specifically, orbitofrontal cortex (OFC) and the ventromedial prefrontal cortex (vmPFC) - the anterior cingulate cortex (ACC), and subcortical structures traditionally associated with the so-called limbic system, particularly the amygdala (Davidson 2000; Quirk and Beer 2006; Ghashghaei 2007). In addition to anatomical connections between the OFC/ACC and the amygdala, functional connectivity analysis has demonstrated that the vmPFC, which has few anatomic connections to the amygdala, is nonetheless functionally coupled to this structure via the ACC, which has direct connections to both (Buckholtz 2008).

Within this group of structures that form the core of the brain's emotional circuitry, the amygdala has primary responsibility for the initial evaluation of emotionally relevant stimuli and their classification, on the basis of prior learning, as positive or negative (Davis 1992; Rolls 1992; Kim 2011). For example, facial expressions of emotion, which convey important information about the motives of others, trigger robust activity in the amygdala (Breiter 1996; Morris 1996, 1998; Patin and Hurlemann 2011). Interactions between the amygdala and the prefrontal cortex refocus attention and engage mechanisms that integrate emotional information with prior learning, self-knowledge, and contextual data, and, as necessary, exert top-down control of the intensity of the response (Quirk and Beer 2006).

Sex differences in the size and activity of the amygdala and prefrontal cortex are well documented. For example, the amygdala of both animals and humans is larger in males (Goldstein 2001; Równiak 2013). In adolescent boys, the larger the left amygdala, the greater the degree of emotional control; emotional control in girls, on the other hand, is associated with a smaller left amygdala (Blanton 2010). A recent study linked male amygdala volume to male violence, reporting that a decrease in the size of the amygdala was associated with an increase in aggression during childhood and adolescence, as well as an increase in violence and psychopathic features as an adult (Pardini 2013). However, it is not known if amygdala volume is altered in violent women as well.

Viewing fearful or angry faces activates the amygdala in both sexes; however, the right amygdala is preferentially activated in men, while the left amygdala is activated in women (Cahill 2004; Andreano and Cahill 2012). Furthermore, the magnitude of responses to angry faces in men has been linked to testosterone levels: men with high testosterone display less activation than those with low testosterone levels, which may indicate that they feel less threatened by the anger of others (Stanton 2009). More research is needed to determine the effect, if any, of these functional differences on social cognition or aggression in the two sexes.
In contrast to the amygdala, the vmPFC and OFC are larger in women (Goldstein 2001; Raine 2011). Research has provided evidence that this difference is directly relevant to sex differences in violence, at least the antisocial form: a 2011 study comparing antisocial behavior, including violence, and OFC volume in men and women calculated that $77.3 \%$ of the difference in behavior between men and women could be accounted for by the smaller size of the OFC in men (Raine 2011).

Brain imaging studies have also documented sex differences in brain activity that suggest that men and women may use their brains differently when exercising self-control or making moral judgments. For example, researchers have identified such differences during a "Go/noGo" task designed to assess impulse inhibition (Liu 2012). Men who successfully refrained from responding to the "noGo" stimulus displayed activation of the ACC that correlated with their impulsivity scores on a personality test. Impulse control in women, on the other hand, was associated with increased activation of the middle temporal cortex. The authors argue that these differences may reflect sex differences in the neural mechanisms governing impulse control-specifically, that activation of the ACC, a region critical to cortical control of responses (Brown 2006), allows men to avoid errors by actively suppressing inappropriate reactions, while in women, activation of the middle temporal cortex, which is involved in focusing attention (Shafritz 2004), may indicate an emphasis on evaluating options and choosing the correct one, rather than subduing the wrong one.

Men and women may also use different strategies to make decisions about moral issues, according to another fMRI study (Harenski 2008). Viewing morally offensive scenes activated the medial prefrontal cortex, posterior cingulate cortex, superior prefrontal cortex, and parts of the parietal cortex in both sexes. However, scenes judged by women to be more egregious triggered more activity in the posterior cingulate cortex, a region linked to the emotional aspect of moral reasoning (Greene and Haidt 2002), and the anterior insula, a region critical to the perception of empathy (Lamm and Singer 2010), consistent with the contention that women put themselves "in the other person's shoes." On the other hand, the scenes men classified as more severe violations were associated with decreased activity in the insula but positively correlated with activity in a part of the parietal cortex near the posterior temporal sulcus, a region involved in executive functions such as reasoning and judgment (Schaich Borg 2006), suggesting that men focus on "the rules."

The existence of sex differences in the neural mechanisms underlying impulse inhibition and moral cognition does not automatically mean that one is necessarily more effective than the other. Nonetheless, additional evaluation of neurocognitive differences is a promising avenue for future research.

\section{Stress: A Different Experience for Men and Women}

Stress is a well-documented example of an environmental factor with adverse biological consequences. Both acute and chronic stress engage not only the brain's emotional circuitry, but also a neuroendocrine cascade, the 
hypothalamic-pituitary-adrenal (HPA) axis, which regulates the secretion of glucocorticoid stress hormones, beginning with the release of corticotropin releasing factor (CRF) by the hypothalamus. CRF triggers the secretion of adrenocorticotropic hormone (ACTH) from the pituitary; $\mathrm{ACTH}$, in turn, acts on the adrenal glands to initiate the release of glucocorticoids. Glucocorticoid receptors in the hypothalamus and pituitary, along with glucocorticoidsensitive neurons in the hippocampus, exercise control over the HPA axis to terminate glucocorticoid secretion. Persistent, extreme, or recurrent stress taxes this feedback regulation and can lead ultimately to abnormalities in HPA axis function that have been linked to a range of psychopathologies (see, e.g., McEwen and Schmeck 1994), including depression, anxiety disorders, hostile violence (in which cortisol levels are elevated), and antisocial/psychopathic violence (associated with reduced cortisol levels and blunted stress responses).

Research on the regulation of the HPA axis has demonstrated that males and females respond differently to stress. Studies in laboratory rodents have consistently found higher basal levels of CRF and corticosterone in females. In addition, stress-induced corticosterone secretion is higher in females and remains elevated longer (Bangasser and Valentino 2012). The picture in humans is more complex. Some studies have also found higher baseline levels of HPA axis activity in women (Bangasser and Valentino 2012). Overall, stress-induced elevations of cortisol tend to be higher in men, but some stressors, such as social rejection, appear to trigger larger cortisol increases in women (Kudielka and Kirshbaum 2005). This raises the interesting possibility that men and women may be differentially sensitive to various forms of stress, and that the likelihood of stress-induced abnormalities in cortisol secretion for both could therefore depend on the risk of exposure to the factors to which each is most sensitive.

As in so many things, timing is everything. There is ample evidence that traumatic stress early in life can "reprogram" the HPA axis in especially disadvantageous and persistent ways (e.g., Van Voorhees and Scarpa 2004; McCrory 2012). Maladaptive changes in stress responses after childhood maltreatment are particularly worrisome given the extensive literature linking childhood abuse and neglect to violent behavior later in life (e.g., Widom and Maxfield 1996; English 2002). If boys are differentially sensitive to the harmful neurobiological effects of abuse, this vulnerability could contribute to sex differences in violence.

\section{Gene-Environment Interactions and Male Vulnerability}

Recent research on the interactions between specific candidate genes and early-life adversity suggests that genetic variation may be an important source of sex-related differences in vulnerability to deleterious environmental factors. The best known and most extensively studied example is the $\mathrm{X}$-linked gene encoding monoamine oxidase A (MAOA), an enzyme responsible for the breakdown of the neurotransmitters norepinephrine and serotonin. As de- scribed below, variants of the promoter region of the $M A O A$ gene lead to higher $(M A O A-H)$ or reduced $(M A O A-L)$ levels of gene expression that have been associated with differences in the brain's emotional circuitry, sensitivity to childhood maltreatment, and violent behavior.

Initial evidence linking the MAOA gene to male aggression came from studies of genetically engineered mice and a Dutch kindred with a rare inherited mutation in the gene. Male $M A O A$ knockout mice are unusually aggressive, have higher levels of norepinephrine and serotonin, and display aberrations in brain development (Buckholtz and MeyerLindenberg 2008). Human males with the MAOA mutation also exhibit an increase in violent behavior, particularly the impulsive outbursts characteristic of hostile violence (Brunner 1993). Subsequent research has focused on comparisons between $M A O A-L$ and MAOA-H genotypes and their relationship to violence.

Brain imaging studies have documented significant reductions in the volume of the amygdala and ACC in both men and women with the $M A O A-L$ genotype, as well as an increase in the activity of the left amygdala and decreased activity in the ACC and OFC in response to angry or fearful faces (Meyer-Lindenberg 2006). Additional alterations in brain structure and function appear specific to $M A O A-L$ men. These include an increase in the volume of the OFC, increased activity in the amygdala during the recall of emotionally aversive information, and reduced activity in the ACC when viewing emotional facial expressions or completing an impulse inhibition task, suggesting a reduction in ACC regulation of the amygdala. Functional connectivity analyses of the two genotypes in response to facial expressions also found a decrease in connectivity between the OFC and amygdala in MAOA- $L$ men, suggestive of an additional deficit in cortical control of amygdala activity (Meyer-Lindenberg 2006). However, a subsequent study reported an increase in functional coupling between the amygdala and the vmPFC - evidence, according to the authors, that may indicate that the $\mathrm{VmPFC}$ is recruited to "shore up" emotional regulation in MAOA-L men as a result of the reduced influence of the OFC and ACC (Buckholtz 2008). This compensatory alteration in a neural mechanism critical to emotional control may represent an underlying vulnerability associated with the MAOA-L genotype, particularly in men.

Research on the interaction between the MAOA gene and childhood maltreatment demonstrates the catastrophic effect of exposing genetically vulnerable individuals to disadvantageous environments. This interaction and its relationship to violence was first documented in the Dunedin Multidisciplinary Health and Development Study, which reported that MAOA-L boys with a history of severe abuse as children were significantly more likely to be diagnosed as young adults with antisocial personality disorder or to be convicted of a violent crime (Caspi 2002). Subsequent studies have replicated the link between the MAOA-L genotype and abuse in the etiology of male antisocial and violent behavior and have extended the association to other stressors, such as neglect and poverty (Kim-Cohen 2006).

The relationship between MAOA gene-environment interactions and violence has been less extensively examined in women, and the results have been less consistent, 
suggesting that the detrimental effect of childhood maltreatment on girls with the MAOA-L genotype may be more restricted or less robust (Sjoberg 2006; Widom and Brzustowicz 2006; Beach 2010). Evidence for the former comes from a recent study that found that the gene-environment interaction in girls was stronger when the analysis was confined to sexual abuse (Beach 2010). In addition, positive environmental influences, such as the support of a nurturing parent, have been shown to moderate the effect of childhood stressors in $M A O A-L$ girls (Kinally 2009). Thus, the $M A O A-L$ genotype is not simply an "accident waiting to happen," but, according to the authors, "may be more aptly described as sensitivity to early-life events." It remains to be seen whether factors such as parental nurturing are equally protective in $M A O A-L$ boys.

\section{Conclusions and Caveats}

More than a decade of research on sex differences in the neural pathways and mechanisms mediating emotional information processing, impulse control, and moral cognition has revealed disparities with intriguing implications for understanding the neurobiology of violence and differences in violent behavior between the two sexes. Nonetheless, many questions remain. One of the most important is the need to better define the relationship between differences at the structural and functional level and differences in behavior. Recent studies demonstrating an association between the volume of the amygdala or OFC and violence represent informative examples. The latter is especially valuable to understanding sex differences because it is one of a minority of studies in which direct comparisons were made between men and women. Brain imaging studies of violent individuals have focused in large part on men, complicating the differentiation of neural features characteristic of violence in both sexes and those specific to violent men.

Observations of sex differences do not resolve the important question of whether such differences are the cause of differences in violent behavior or the consequence of behaving violently. This issue highlights the urgent need for more longitudinal studies. Sex differences in the trajectory of brain development have also been observed and further reinforce the need for such research to avoid confusing age differences and true sex differences. This issue is especially relevant to studies of the prefrontal cortex, one of the last brain regions to mature in both sexes (Gogtay 2004).

Studies of the interaction between the MAOA gene and abuse illustrate not only the collaboration between genes and environment, but also the way in which social experiences become physical realities. This work suggests that one important source of male violence may be male vulnerability, a product of genetic risk factors and the sociocultural environment experienced by boys, including possible sexspecific differences in exposure to various forms of stress, abuse, and other environmental variables. Research that furthers our understanding of the complex interdependence of genetic and environmental factors in the development of violence in both sexes is therefore one of our most pressing priorities.

The increased risk of violence in genetically vulnerable individuals after childhood maltreatment and other forms of early-life adversity also suggests that the first years of life represent a critical period in the development of the neural circuitry underlying emotion and its regulation. As a result, interventions that target early childhood, particularly those that address the needs of at-risk parents; teach effective parenting strategies; promote the recovery of stress-response mechanisms in abused children; and enhance resilience, such as home visits by nurses (Olds 1998) and parent skills training (Webster-Stratton and Taylor 2001), are likely to prove especially beneficial to children with risk-conferring genotypes. The demonstration of a protective effect of supportive parenting in $M A O A-L$ girls exposed to stressors, including abuse, is promising in this regard.

Overinterpretation and oversimplification are two pitfalls that compromise the critical appraisal of neuroscience research, including research on sex differences. In particular, the temptation to merge results to create a composite picture of "male" and "female" brains, while powerful, should be resisted. The differences described here represent differences between group averages; however, overlap between the two sexes is the rule rather than the exception. In addition, differences in individual parameters - the volume of the amygdala and a pattern of brain activation during impulse inhibition-tell us little about whether an individual who has an amygdala similar in size to the male group average also displays the "typical" male pattern of activation during a Go/noGo task. In fact, it is more likely, in the words of psychologist Daphna Joel, that "different individuals will have different combinations of 'male' and 'female' brain characteristics. In this sense, brains are neither 'male' nor 'female', they are intersex"' (Joel 2011).

The neurobiological correlates of violent behavior are not limited to interactions between the prefrontal cortex and amygdala, the mechanisms regulating stress hormones, or a single gene. Violence has polygenetic and multifactorial origins, involving contributions from interconnected networks of brain regions, multiple chemical signals (both neurotransmitters and hormones), and multiple genes, all of which can be altered by a wide range of environmental factors. Ongoing research to identify these factors and their interactions promises to further elucidate the etiology of violent behavior and, ultimately, lead to effective, biologically based intervention and prevention strategies.

\section{Author Disclosure Statement}

No competing financial interests exist.

\section{References}

Andreano JM, Cahill L. (2012). Sex influences on the neurobiology of learning and memory. Learn Mem. 16, 248-266.

Bangasser DA, Valentino RJ. (2012). Sex differences in molecular and cellular substrates of stress. Cell Mol Neurobiol. 32, 709-723.

Beach SRH, Brody GH, Gunter TD, et al. (2010). Child maltreatment moderates the association of MAOA with symptoms of depression and antisocial personality disorder. J Fam Psychol. 24, 12-20.

Bennett S, Farrington DP, Huesmann LR. (2005). Explaining gender differences in crime and violence: The importance of social cognitive skills. Aggress Violent Behav. 10, 263-288.

Blanton RE, Chaplin TM, Sinha R. (2010). Sex differences in the correlation of emotional control and amygdala volumes in adolescents. Neuroreport. 21, 953-957. 
Breiter HC, Etcoff NL, Whalen PJ, et al. (1996). Responses and habituation of the human amygdala during visual processing of facial emotion. Neuron. 17, 875-887.

Brown SM, Manuck SB, Flory JD, et al. (2006). Neural basis of individual differences in impulsivity: Contributions of corticolimbic circuits for behavioral arousal and control. Emotion. 6, 239-245.

Brunner HG, Nelen M, Breakefield A, et al. (1993). Abnormal behavior associated with a point mutation in the structural gene for monoamine oxidase. Science. 262, 578-580.

Buckholtz JW, Meyer-Lindenberg A. (2008). MAOA and the neurogenetic architecture of human aggression. Trends Neurosci. 31, $120-129$.

Buckholtz JW, Callicott JH, Kolachana B, et al. (2008). Genetic variation in MAOA modulates ventromedial prefrontal circuitry mediating individual differences in human personality. Mol Psychiatry. 13, 313-324.

Cahill L, Uncapher M, Kilpatrick L, et al. (2004). Sex-related hemispheric lateralization of amygdala function in emotionally influenced memory: An fMRI investigation. Learn Mem. 11, 261-266.

Caspi A, McClay J, Moffitt TE, et al. (2002). Role of genotype in the cycle of violence in maltreated children. Science. 297, 851-854.

Coccaro EF, McCloskey MS, Fitzgerald DA, et al. (2007). Amygdala and orbitofrontal reactivity to social threat in individuals with impulsive aggression. Biol Psychiatry. 62, 168-178.

Curley JP, Mashoodh R, Champagne FA. (2011). Social influences on neurobiology and behavior: Epigenetic effects during development. Psychoneuroendocrinology. 36, 352-371.

Davidson RJ, Putnam KM, Larson CL. (2000). Dysfunction in the neural circuitry of emotion regulation - a possible prelude to violence. Science. 289, 591-594.

Davis M. (1992). The role of the amygdala in fear and anxiety. Annu Rev Neurosci. 15, 353-375.

English DJ, Widom CS, Brandford C. (2002). Childhood victimization and delinquency, adult criminality, and violent criminal behavior: A replication and extension. Final report presented to the National Institute of Justice. Grant No. 97-IJ-CX-0017.

Ghashghaei HT, Hilgetag CC, Barbas H. (2007). Sequence of information processing for emotions based on the anatomic dialogue between prefrontal cortex and amygdala. Neuroimage. 34, 905-923.

Glenn AL, Raine A. (2008). The neurobiology of psychopathy. Psychiatr Clin North Am. 31, 463-475.

Gogtay N, Giedd JN, Lusk L, et al. (2004). Dynamic mapping of human cortical development during childhood through early adulthood. Proc Natl Acad Sci USA. 101, 8174-8179.

Goldstein JM, Seidman LJ, Horton NJ, et al. (2001). Normal sexual dimorphism of the adult human brain assessed by in vivo magnetic resonance imaging. Cerebral Cortex. 11, 490-497.

Greene J, Haidt J. (2002). How (and where) does moral judgment work? Trends Cogn Sci. 6, 517-523.

Haller J, Mikics É, Halász J, et al. (2005). Mechanisms differentiating normal from abnormal aggression: Glucocorticoids and serotonin. Eur J Pharmacol. 526, 89-100.

Harenski CL, Antonenko O, Shane MS, et al. (2008). Gender differences in neural mechanisms underlying moral sensitivity. Scan. 3, 33-41.

Joel D. (2011). Male or female? Brains are intersex. Fron Integr Neurosci. 5, 1-5.

Kim MJ, Loucks RA, Palmer AL, et al. (2011). The structural and functional connectivity of the amygdala: From normal emotion to pathological anxiety. Behav Brain Res. 223, 403-410.

Kim-Cohen J, Caspi A, Taylor A, et al. (2006). MAOA, maltreatment, and gene-environment interaction predicting children's mental health: New evidence and a meta-analysis. Mol Psychiatry. 11, 903-913.

Kinally EL, Huang Y, Haverly R, et al. (2009). Parental care moderates the influence of MAOA-uVNTR genotype and childhood stressors on trait impulsivity and aggression in adult women. Psychiatr Genet. 19, 126-133.

Kudielka BM, Kirschbaum C. (2005). Sex differences in HPA axis response to stress: A review. Biol Psychiatry. 69, 113-132.

Lamm C, Singer T. (2010). The role of the anterior insular cortex in social emotions. Brain Struct Funct. 214, 579-591.

Liu J, Zubieta J-K, Heitzeg M. (2012). Sex differences in anterior cingulate cortex activation during impulse inhibition and behavioral correlates. Psychiatry Res. 201, 54-62.

McCrory E, De Brito SA, Viding E. (2012). The link between child abuse and psychopathology: A review of neurobiological and genetic research. J R Soc Med. 105, 151-156.

McEwen BS, Schmeck HM. (1994). The Hostage Brain. (The Rockefeller University Press, New York, NY.)

Meyer-Lindenberg A, Buckholtz JW, Kolachana B, et al. (2006). Neural mechanisms of genetic risk for impulsivity and violence in humans. Proc Natl Acad Sci USA. 103, 6269-6274.

Moffitt TE, Caspi A, Rutter M, et al. (2001). Sex differences in antisocial behavior: Conduct disorder, delinquency, and violence in the Dunedin Longitudinal Study. (Cambridge University Press, Cambridge.)

Morris JS, Frith CD, Perrett DI, et al. (1996). A differential neural response in the human amygdala to fearful and happy facial expressions. Nature. 383, 812-815.

Morris JS, Öhman A, Dolan RJ. (1998). Conscious and unconscious emotional learning in the human amygdala. Nature. 393, 467-470.

Niehoff DL. (1999). The Biology of Violence: How Understanding the Brain, Behavior, and Environment Can Break the Vicious Circle of Aggression. (The Free Press, New York, NY.)

Olds D, Henderson CR Jr., Cole R, et al. (1998). Long-term effect of nurse home visitation on children's criminal and antisocial behavior: 15-year follow-up of a randomized controlled trial. JAMA. 282, 479-481.

Pardini DA, Raine A, Erikson K, et al. (2013). Lower amygdala volume in men is associated with childhood aggression, early psychopathic traits, and future violence. Biol Psychiatry. DOI:10.1016/ j.biopsych.2013.04.003. [Epub ahead of print]

Patin A, Hurlemann R. (2011). Modulating amygdala responses to emotion: Evidence from pharmacological fMRI. Neuropsychologia. 49, 706-711.

Quirk GJ, Beer JS. (2006). Prefrontal involvement in the regulation of emotion: Convergence of rat and human studies. Curr Opin Neurobiol. 16, 723-727.

Raine A, Yang Y, Narr KL, et al. (2011). Sex differences in orbitofrontal gray as a partial explanation for sex differences in antisocial personality. Mol Psychiatry. 16, 227-236.

Rolls ET. (1992). Neurophysiology and functions of the primate amygdala. In The Amygdala: Neurobiological Aspects of Emotion, Memory, and Mental Dysfunction. Aggleton JP, ed. (Wiley-Liss, New York, NY), pp 143-165.

Równiak M. (2013). The amygdala in the guinea pig is sexually dimorphic - a morphometric study. Brain Res. 1524, 44-53.

Schaich Borg J, Hynes C, Van Horn J, et al. (2006). Consequences, action, and intention as factors in moral judgments: An fMRI investigation. J Cog Neurosci. 18, 803-817.

Shafritz KM, Marchione KE, Gore JC, et al. (2004). The effects of methylphenidate on neural systems of attention in attention deficit hyperactivity disorder. Am J Psychiatry. 161, 1990-1997.

Sjoberg RL, Nilsson KW, Wargelius HL. (2006). Adolescent girls and criminal activity: Role of MAOA-LPR genotype and psychosocial factors. Am J Med Genet B Neuropsychiatr Genet. 144, 159-164.

Stanton SJ, Wirth MM, Waugh CE, et al. (2009). Endogenous testosterone levels are associated with amygdala and ventromedial prefrontal cortex responses to anger faces in men but not women. Biol Psychiatry. 81, 118-122. 
Stevenson RA. (2012). Using functional connectivity analyses to investigate the bases of autism spectrum disorders and other clinical populations. J Neurosci. 32, 17933-17934.

Van Voorhees E, Scarpa A. (2004). The effects of child maltreatment on the hypothalamic-pituitary-adrenal axis. Trauma Violence Abuse. 5, 333-352.

Webster-Stratton C, Taylor T. (2001). Nipping early risk factors in the bud: Preventing substance abuse, delinquency, and violence in adolescence through interventions targeting young children (0-8 years). Prev Sci. 2, 165-192.

Widom C, Brzustowicz L. (2006). MAOA and the cycle of violence: Childhood abuse and neglect, MAOA genotype, and risk for violent and antisocial behavior. Biol Psychiatry. 60, 684-689.
Widom CS, Maxfield MG. (1996). A prospective examination of risk for violence among abused and neglected children. Ann NY Acad Sci. 794, 224-237.

Address correspondence to: Debra Niehoff, PhD Department of Social and Behavioral Science Bucks County Community College 275 Swamp Road Newtown, PA 18940

E-mail: debra.niehoff@bucks.edu 